\title{
Tumor Identification Time
}

National Cancer Institute

\section{Source}

National Cancer Institute. Tumor Identification Time. NCI Thesaurus. Code C162179.

The time of tumor identification data collection. 